\title{
Don't Text While Driving: The Effect of Smartphone Text Messaging on Road Safety during Simulated Driving
}

\author{
Kaspar Lyngsie $^{1,2}$, Martin S. Pedersen ${ }^{1,3}$, Jan Stage ${ }^{1}$, and Kim F. Vestergaard ${ }^{1,4}$ \\ ${ }^{1}$ Aalborg University, Department of Computer Science, 9220 Aalborg, Denmark \\ ${ }^{2}$ Gamblify, Lyngbyvej 21, 2100 Copenhagen, Denmark \\ ${ }^{3}$ Telenor, Skelagervej 9, 9000 Aalborg, Denmark \\ ${ }^{4}$ Danske Bank, Edwin Rahrsvej 40, 8220 Brabrand, Denmark \\ kml@gamblify.com, novacdk@gmail.com, \\ jans@cs.aau.dk, kive@danskebank.dk
}

\begin{abstract}
Text messaging on smartphones uses a full soft keyboard instead of the numeric buttons on traditional mobile phones. While being more intuitive, the lack of tactile feedback from physical buttons increases the need for user focus, which may compromise safety in certain settings. This paper reports from an empirical study of the effect of text messaging on road safety. We compared the use of a traditional mobile phone and a smartphone for writing text messages during simulated driving. The results confirm that driver performance when texting decreases considerably as there are significant increases in reaction time, car-following distance, lane violation, number of crash/near-crash incidents, perceived task load and the amount of time the driver is looking away from the road. The results also show that smartphones makes this even worse; on key performance parameters they increase the threat from text messaging while driving. These results suggest that drivers should never text while driving, especially not with a smartphone.
\end{abstract}

Keywords: Driving, mobile phone, smartphone, text messaging, road safety, driver distraction, simulated driving experiment.

\section{Introduction}

Smartphone adoption is accelerating. In 2010, about $20 \%$ of the mobile phones sold were smartphones, and for 24-35 year olds $31 \%$ [16, 30]. A recent market analysis expected smartphone adoption to surpass that of traditional mobile phones by the end of 2011 [13]. The new smartphones provide more functionality, and touch-based interaction on a larger screen facilitates improvement of many functions.

Text messaging is a widely used form of communication on mobile phones. Early systems used the Multitap technique, where a letter is entered by pressing a numerical button a number of times. Later, Multitap was replaced by the predictive text entry system known as T9, which also relies on the numerical buttons but reduces the number of key taps and, thereby, allows text writing at the double speed [11]. On modern smartphones, text messages are written on a full QWERTY keyboard on the touch 
screen. This is obviously more intuitive for writing text messages than Multitap and T9. Yet the physical buttons on traditional mobile phones offer a tactile feedback that is lacking on the touch screen of a smartphone. This lack of tactile feedback may be a serious problem in special situations.

The number of drivers that are text messaging while driving has increased tremendously. A recent survey showed that as many as $51 \%$ of all young drivers admitted to have used text messaging during driving [15]. Up to one quarter of all car crashes are estimated to be a result of drivers engaging in distracting activities [19, 24]. Use of mobile phones while driving has a range of negative effects, including distracting the driver [26, 28, 36].

While the consequences of using mobile phones for calling and having conversations have been studied thoroughly, there are much fewer studies of the hazards of text messaging. Two recent studies found that text messaging while driving increases the risk of being in a crash or near-crash by 23 times [7, 16]. Unfortunately, the few studies that have been made are based on traditional mobile phones with tactile feedback $[19,23]$. So far, it has not been documented how use of touch-based smartphones for texting while driving influence road safety.

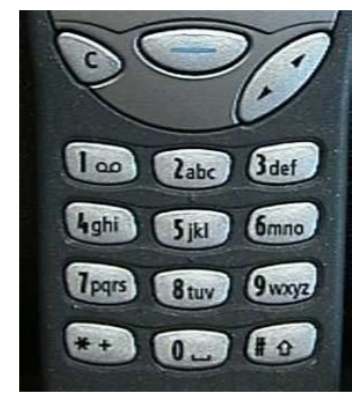

Fig. 1. The keypad layout on a Nokia 3210

This paper reports from an empirical study of the effect of text messaging on road safety. The aim was to inquire into the safety hazards when texting on a smartphone while driving compared to a more traditional mobile telephone. In the following section, we describe text entry methods on mobile phones. Then we provide an overview of related work. The next section presents the method of our experiment that was carried out in a driving simulator. Then we present the results where we compare the two types of phones used for texting while driving. Next, the results are discussed and compared to related work. Finally, we provide the conclusion.

\section{Text Entry Methods}

The first text messaging systems, originally denoted as Short Message System or SMS, used the numerical keys on the mobile phone for text entry. The letters of the 
alphabet were distributed with more than one letter on each physical button, see Figure 1. The layout is a grid of 12 buttons, where the alphabet is distributed on buttons 2-9.

The Multitap technique was the first widely used implementation of text entry with this layout. With the Multitap technique, the user taps one or more times on a button to get a letter. To write the word 'hey', you need to press twice on button 4 to get ' $h$ ', twice on 3 to get ' $e$ ' and three times on 9 to get ' $y$ '. There is a timeout period of usually $0.5-2$ seconds after each key press, which is used to delimit letter selections on the same button. So to write the word 'hi', you would need to press two times on 4 to get ' $h$ ', wait for the timeout period and then press three times on 4 to get ' $I$ '. The number of words that can be typed per minute with Multitap is about 5-10 wpm depending on experience [20].

In the late 1990s the "text on nine keys" (T9) predictive text entry technique was developed by Tegic Communications. It was based on the same keyboard layout as Multitap, but it used dictionaries to predict the desired text entry, based on the buttons being pressed. The T9 technique needs only one press on each button to predict what word is being written. To write the word 'hey', you only need to press the buttons 4, 3 and 9 to have the software suggest the word 'hey' and potentially other typical words that could be composed by tapping the same three buttons. The most frequent used word will be predicted first. T9 was quickly adopted as the primary form of text entry on products from all the major mobile phone manufacturing companies [11]. The number of words that can be typed per minute with T9 is about 7-25 wpm depending on experience [20], which is about twice the speed as with Multitap.

With the introduction of modern smartphones, the use of a mobile phone has exceeded that of simple telephony and text messaging, and the 9-button interface has been replaced by virtual full-size keyboards on touch screens. Smartphones are now also used for more advanced features such as document editing and mail composition and research are continuously attempting to increase the efficiency of the limited space available for text entry.

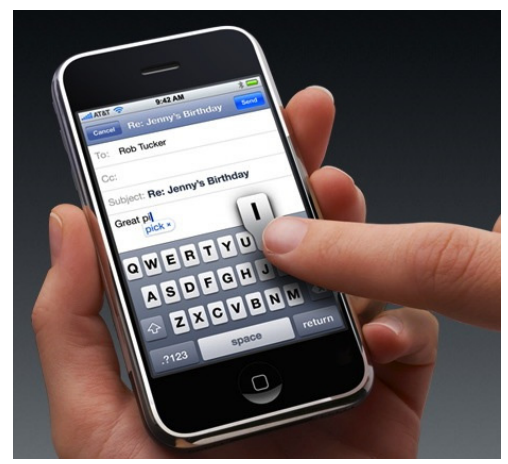

Fig. 2. Writing text on an Apple iPhone

Opposed to the common feature phones, most smartphones require larger screens in order to accommodate for the use of more advanced appliances such as email 
composition and text edition. This allows the smartphones such as Apple iPhone and many HTC Android-based phones to remove the physical keyboard all together and instead use an on-screen, full-size QWERTY keyboard which is stimulated through touch.

Writing text on a typical on-screen keyboard such as the Apple iPhone, see Figure 2, is done by touching the keyboard character on the screen. This solution also use dictionaries to predict the intended word, but does so by considering the surrounding characters for each key press [25]. A study on the Apple iPhone touch-based smartphone concluded the text entry speed to be $15.9 \mathrm{wpm}$ [5].

\section{Related Work}

There is a broad variety of literature on mobile phone use while driving. Some of the literature emphasize the potential problems, e.g. that visual processing is affected by checking to see who is calling, motor control is affected when dialling a number, auditory distraction when attention is given to the conversation and not the sound of the traffic and higher-order (cognitive) processing when focusing on the conversation and not the act of driving [22]. There are also discussions where notable accidents with multiple casualties have been attributed to texting while driving [14].

Statistics show that teenagers are clearly the age group with the highest risk of being involved in accidents. Studies show that for every mile driven in the United States, teenagers are four times more likely to be involved in a car crash [2]. Studies on text-messaging habits in the last decade show an increase from 12 million to 135 billion text messages sent every month, where teenagers clearly being the most active age group, sending and receiving an average of 3,000 messages per month $[6,10]$. Surveys conducted among teenagers conclude that half of all students admit to having texted while driving [19].

Table 1. Overview of related publications on mobile phone use while driving either in real world traffic or a car simulator

\begin{tabular}{|l|c|c|c|c|}
\hline & \multirow{3}{*}{ Dialling } & \multicolumn{3}{|c|}{ Mobile Phone Task } \\
& & Talking & Texting \\
& & $7,8,31$ & 8,27 & 7 \\
\hline Real world & 7,32 & $7,-h e l d$ & Hands-free & \\
\hline Simu-lator & 28,32 & $9,28,35$ & $3,4,35$ & 34 \\
\hline
\end{tabular}

A significant number of empirical studies have inquired into the effects of mobile phone use on road safety, see Table 1. An early study, based on simulated driving, tried to establish the dangers when dialling and talking on a mobile phone [28]. 
A study of overall driving performance based on a car simulator concluded that a person talking on a mobile phone was more prone to being involved in a crash or near-crash event than a drunk driver [35]. A recent study based on a simulator concluded that the use of a mobile phone was much more distracting than a conversation with a passenger [9]. This confirms an early study in real world traffic where it was concluded that there is a much smaller risk when engaging in conversations with the passenger than talking on a mobile phone [8].

Many countries around the world have banned the use of hand-held mobile phones while driving. Yet an early study in a simulator concluded that a hands-free mobile phone was also dangerous to use while driving [3,4]. This is confirmed by more recent studies in real traffic establishing that the risks of using a hands-free mobile phone are the same as using a hand-held, e.g. [27, 32]. The reason is that most of the distraction of talking on the phone is not related to visual processing or motor control, but to the cognitive workload.

Despite the dangers of hand-held and hands-free mobile phone usage, only very few countries have banned both, where more countries have customized laws depending on age group of the drivers or the location of where the driving is conducted [1]. Much debate has been raised about the effectiveness of prohibition, as the task of actually enforcing these laws has proven to be very difficult [17].

Studies conducted in real-world scenarios are harder to conduct, but have shown results that are similar to the car simulator studies. One study used accident analysis, where drivers who had been involved in car accidents were questioned about their mobile phone use prior to the accident, and their statements were compared to data from their mobile phone providers. The results showed that almost a quarter of these individuals had used their mobile phone in the 10 minutes preceding the crash [31].

In recent real world studies, the dangers associated with mobile phone usage has been quantified in more detail, where actions such as dialling a mobile phone increases the risk of accidents by 6 times and talking and listening double the risk [7].

Only few studies have been conducted on the subject of text messaging while driving. A notable exception is a study from Virginia Tech in 2009 where they observed drivers for more than 6 million miles of driving. It was concluded that texting while driving increased the risk of being involved in an accident by 23 times [7]. Most surprisingly, there is only a single study of text messaging on a smartphone, even though a market share analysis expects touch-based smartphone adoption to surpass that of feature phones by the end of 2011 [13]. In this single study, they inquired into texting on a Blackberry and an iPhone during simulated driving. Their focus was on the reasons why the number of texting car crashes is different and on differences between frequent and less frequent texters [34]. Thus they did not compare smartphones and traditional mobile telephones.

There are also a number of studies of the effects of interaction with other types of touch-based devices with while driving. One example is a study of GPS navigator use during real driving where they showed that the attention on visual output decreased driver performance significantly [21]. Another example is a study of the distraction caused by iPod use during simulated driving where they concluded that the effect was similar to that of operating a mobile phone [33]. 


\section{$4 \quad$ Method}

This section describes the experiment we conducted to study the effect on road safety when using a traditional mobile phone and a smartphone for writing text messages during simulated driving.

\subsection{Setting}

The study was conducted in a car simulator that was set up in the usability laboratory at HCI-Lab, Department of Computer Science, Aalborg University.

\subsection{Apparatus}

The car simulator was based on a computer with three screens, see Figure 3 . The screen used for the front view of the road ahead was a 37" screen, and the side-view windows were two 32 " screens. The screens were controlled by a powerful computer with extra graphics cards and a device for splitting the main display to the three screens.

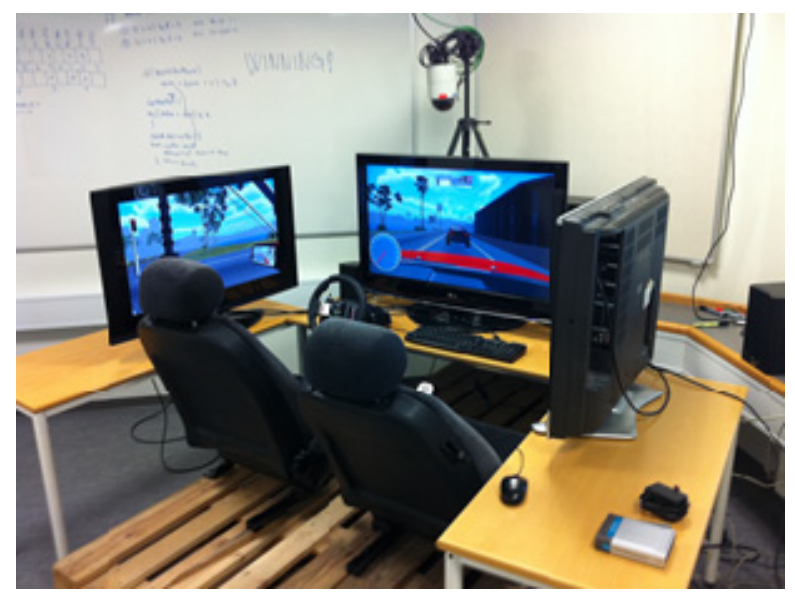

Fig. 3. The physical setup of the simulator

The physical setup was centred around a passenger and a driver's seat. A standard driving kit was used to control the simulator. The kit included a steering wheel, pedal board with gas, brake and clutch pedals and a manual gear shift. Tactile transducers called "bass shakers" were added to the driver's seat to simulate engine vibration.

The software for the simulator was created using the Unity 3D game engine and the virtual world was modelled using CityScape. The car physics was based on an open source tutorial that was extended with support for the driving kit. A new module was developed to simulate traffic, and the city was fitted with intersections and traffic lights. A logger module recorded reaction times, velocity, distance from the middle of the lane etc. 


\subsection{Participants}

The group of test subjects consisted of 28 people, where 18 were male and 10 were female. Among them, 10 normally used a T9 mobile phone and 18 normally used a touch-based smartphone. The participants all held valid driver's licenses and were in the age of 20-32 years old and had a variety of occupations.

\subsection{Procedure}

The subjects were instructed about the experiment. Then they made a test-drive in the car simulator in a city environment to get acquainted with the controls and behaviour of the car. No measurements were made during this drive.

Two driving scenarios were used: city and freeway. The freeway was a straight road with three lanes, a higher speed limit and traffic going at various speeds. The city had curved roads, intersections, traffic lights, a hilly terrain and traffic.

The experiment was conducted as between-subjects, where half of the subjects drove on the freeway, and the other half drove in the city. The participants were balanced over these two scenarios according to gender and mobile phone experience.

In each scenario, the subjects started in a baseline condition, which was a 5-minute drive without interacting with a mobile phone. Then they drove in the same scenario using one of the two mobile phones. Finally, they drove in the other scenario using the other mobile phone. The mobile phones were a touch-based smartphone and a tactile T9 phone. The uses of the two mobile phones were balanced in each scenario between test subjects in order to avoid a learning effect.

In the two conditions with mobile phones, the test subjects were instructed to enter up to five different text messages with a time limit of 5 minutes. If they did not complete all five messages during the 5-minute drive, the remaining messages were registered as in-complete. They were not required to complete the 5 messages. During the drive, the test monitor would read out the first sentence they had to write. When they had completed the sentence, they stated that, and the test monitor would read the next message and so on until they had written the five messages or the five minutes had passed. All text messages were randomly selected sentences of the same length and complexity [25] which were distributed among the subjects using latin squares. An example of a sentence is: "we are coming back tomorrow morning".

In all three conditions, the test subjects had to follow a car driving in front of them. The car in front was programmed to brake at random intervals and it would continue to break until the test subject either pressed the brake pedal or collided with it. This is a commonly used way to measure the test subjects' reaction time in simulated driving [11].

After each condition, the test subjects filled in NASA Task Load Index (TLX)-scales, which is a NASA developed method for measuring task load [18]. 


\subsection{Data Collection}

Two cameras were used to record video: one focused on the test subject's eyes for eye glance recording and the other was focused directly down on the subject's interaction with the phone. These two images were mixed together with the front-view screen (the side-view screens were not recorded) to produce the material which was recorded on DVD and later analysed, see Figure 4.

The simulator was programmed to create log files for each condition. These log files included values such as distance to the centre of the lane, distance to the followed car, velocity, user reaction time, crashes and task completion times.

\subsection{Data Analysis}

Two aspects were analysed manually from the video recordings: near-crash incidents and eye glances.

Crashes and near-crashes are often used as a measure in the literature on driver performance. The simulator software was programmed to log crashes, while near-crashes had to be analysed manually.

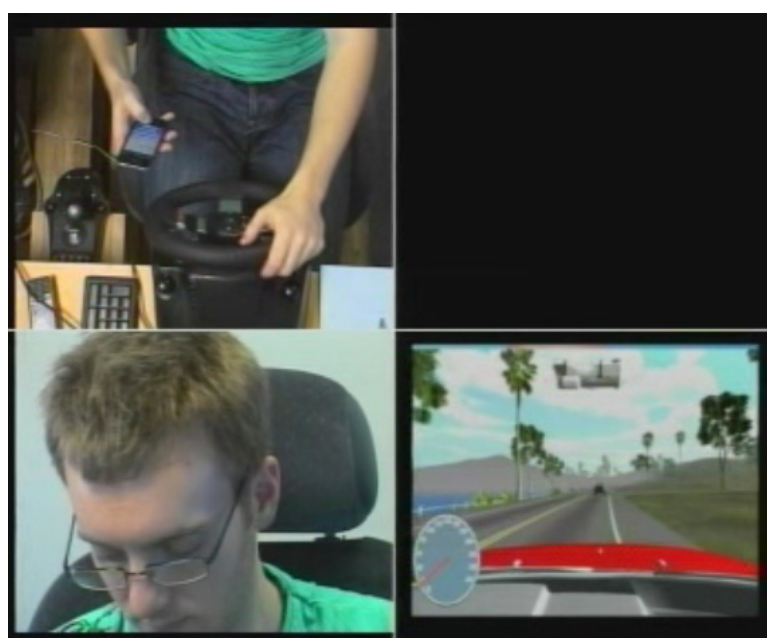

Fig. 4. A subject making an eye glance while typing on a smartphone

For the purpose of this analysis, we used the related literature to define a near-crash as an incident where the driver was very close to crashing or only narrowly avoided a collision with the lead car or another car or object. For example, if the driver came into the opposite lane and only in the last fraction of a second avoided a head-on collision; or if the driver had to emergency brake to avoid the car he was following.

For eye glances, there was an error in the video recording for one test subject which implied that the eye glances could not be detected. Therefore, the data on eye glances are only from 27 test subjects. The one missing is from the city condition. The 27 recordings were analysed frame by frame for eye glances. The frame where the 
subject's eyes started moving away from the front window was registered as the first frame in the eye glance, and the frame before the first frame where the eyes were back on the front window was registered as the end of the eye glance. The length of each eye glance was calculated from the number of frames (it was recorded as $24 \mathrm{fps}$ ). This was done separately by three of the authors. After the analysis, they compared their results. The disagreements were mostly a couple of frames in the beginning or end of an eye glance. We calculated the Fleiss Kappa for the three raters across all eye glances. The result was Kappa $=0.844$, which is categorized as almost perfect agreement.

The NASA TLX answers and weights were grouped as prescribed by the manual [18]. The data from the driving scenarios was cleaned manually and organized using software developed specifically for this task and then imported for statistical analysis in the R statistical software environment.

We performed repeated measures ANOVA with condition as the repeated factor and road type as a between-subject factor.

\section{$5 \quad$ Results}

This section contains the results from the experiment, grouped into driving performance, task performance and eye glances.

Table 2. Means and standard deviations of driving performance

\begin{tabular}{l|lll} 
& \multicolumn{3}{|c}{ Condition } \\
\hline Variable & Baseline & T9 & Touch \\
\hline City & & & \\
Reaction time (ms) & $1206(454)$ & $2200(1104)$ & $2363(720)$ \\
Crash or near-crash & $0.36(0.50)$ & $1.36(1.39)$ & $1.71(1.38)$ \\
Following distance (m) & $23.52(6.52)$ & $29.30(6.62)$ & $29.21(69.44)$ \\
Following distance variability & $10.66(3.42)$ & $14.09(3.85)$ & $14.43(4.14)$ \\
Lane crossings per kilometer & $8.98(1.46)$ & $9.58(2.92)$ & $10.08(2.27)$ \\
Time in wrong lane per kilometer (s) & $48.60(20.14)$ & $82.23(46.70)$ & $97.40(37.05)$ \\
Lane variability & $1.79(0.12)$ & $1.61(0.36)$ & $1.71(0.25)$ \\
& & & \\
Freeway & & & \\
Reaction time (ms) & $1281(397)$ & $2723(1181)$ & $2568(908)$ \\
Crash or near-crash & $0.64(1.00)$ & $1.14(0.86)$ & $2.43(2.24)$ \\
Following distance (m) & $32.50(8.39)$ & $43.73(14.14)$ & $42.85(12.18)$ \\
Following distance variability & $12.55(4.08)$ & $23.59(9.41)$ & $23.42(8.60)$ \\
Lane crossings per kilometer & $0.00(0.00)$ & $0.22(0.56)$ & $0.81(1.03)$ \\
Time in wrong lane per kilometer (s) & $0.00(0.00)$ & $4.027(10.45)$ & $10.73(20.21$ \\
Lane variability & $0.27(0.07)$ & $0.45(0.17)$ & $0.57(0.30)$ \\
& & & \\
\hline
\end{tabular}




\subsection{Driving Performance}

The results for driving performance are shown in Table 2.

Reaction Time. The reaction time was approximately doubled, an increase of a little more than one second, when writing text messages, and this difference is significant both on the freeway $(\mathrm{F}=21.4, \mathrm{p}<0.001)$ and in the city $(\mathrm{F}=17.6, \mathrm{p}<0.001)$.

Assuming the subject is driving at $50 \mathrm{~km} / \mathrm{h}$, as the speed limit was in the city scenario, one second of increased reaction time would translate to an extra 13 meters of breaking distance.

There was no significant difference in reaction time between the Touch and T9 conditions. The analysis showed no significant differences in reaction time when driving in the city and on the freeway.

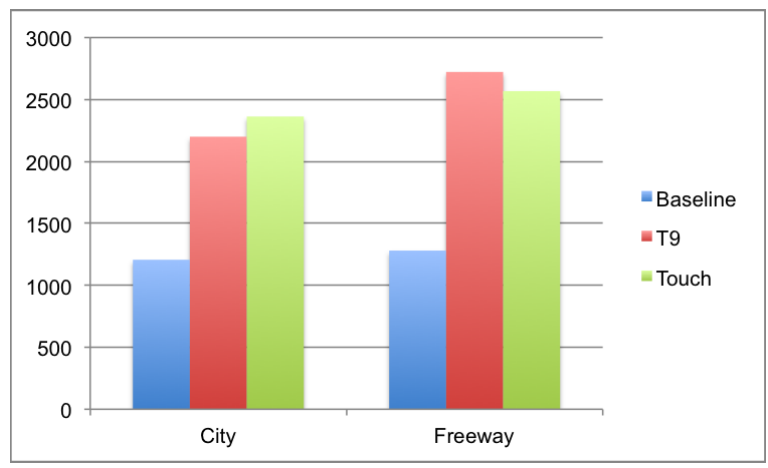

Fig. 5. Reaction time

Crash or Near-Crash. The number of crashes and near-crashes increased significantly between the baseline condition and the writing conditions both in the city $(\mathrm{F}=6.40$, $\mathrm{p}<0.01)$ and on the freeway $(\mathrm{F}=5.13, \mathrm{p}<0.05)$. There were significantly more crashes or near-crashes when writing with Touch than with $\mathrm{T} 9$ on the freeway $(\mathrm{F}=5.92, \mathrm{p}<0.05)$. There is a similar tendency in the city, but this difference is not significant.

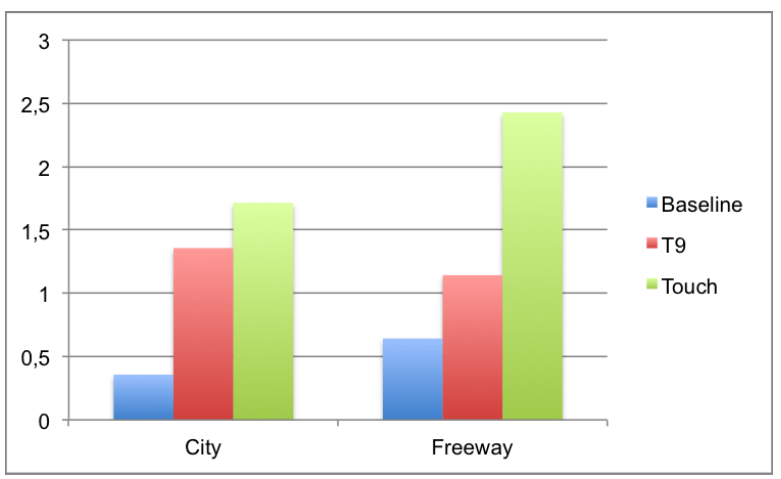

Fig. 6. Crash or near-crash 
Following Distance. The average following distance shown in Table 2 reveals that the participants increased their distance to the car in front of them when they were writing text messages. The analysis showed significant difference between the baseline condition and the writing conditions both on the freeway $(\mathrm{F}=11.64, \mathrm{p}<0.001)$ and in the city $(\mathrm{F}=11.59, \mathrm{p}<0.001)$.

The variability in following distance increased significantly when the participants were writing text messages compared to the baseline condition both on the freeway $(\mathrm{F}=20.13, \mathrm{p}<0.001)$ and in the city $(\mathrm{F}=11.04, \mathrm{p}<0.001)$.

There were no significant differences in following distance or following distance variability between Touch and $\mathrm{T} 9$ conditions.

Lane Maintenance. The average lane crossings per kilometer increased in the writing conditions, but the increase was only significant on the freeway $(\mathrm{F}=6.28, \mathrm{p}<0.01)$. The Touch condition had more lane crossings per kilometer than the T9 condition, and this difference was only significant on the freeway $(\mathrm{F}=5.66, \mathrm{p}<0.05)$.

The difference in the time spent in the wrong lane between the baseline condition and the writing conditions is significant in the city $(\mathrm{F}=16.25, \mathrm{p}<0.001)$, but not on the freeway. This means that although the number of lane crossings in the city is not significantly higher when writing, the time to correct and get the car back in the correct lane is longer.

The time spent in the wrong lane is also higher in the Touch condition than in the T9 condition, but this difference is not significant.

The variability within the lane increased when writing on the freeway, and this increase was significant $(\mathrm{F}=8.66, \mathrm{p}<0.01)$. No such increase was found in the city, and there was no significant difference in lane variability between Touch and T9 conditions.

The lane variability indicates a severe change in driving behaviour, and swerving into another lane increases the risk of the driver being involved in a crash or nearcrash situation. This risk increases the more time the driver spends in the wrong lane.

Table 3. Means and standard deviations of task performance

\begin{tabular}{l|lcc} 
& \multicolumn{3}{|c}{ Condition } \\
\hline Variable & Baseline & T9 & Touch \\
\hline City & & $52.67(21.04)$ & $58.19(20.89)$ \\
Task load & $26.42(12.55)$ & $3.86(1.56)$ & $3.79(1.58)$ \\
Task completions & N/A & $38.17(31.85)$ & $44.87(25.93)$ \\
Task completion time (s) & N/A & $61.91(24.92)$ & $49.84(26.59)$ \\
Charactersper minute & N/A & & \\
\hline Freeway & & & \\
Task load & 23.79 (15.29) & $45.54(26.42)$ & $53.77(26.89)$ \\
Task completions & N/A & $4.64(0.74)$ & $4.00(1.66)$ \\
Task completion time (s) & N/A & $30.23(8.85)$ & $44.61(29.34)$ \\
Charactersper minute & N/A & $53.55(16.85)$ & $47.14(22.43)$ \\
\hline
\end{tabular}




\subsection{Task Performance}

The results for task performance are shown in Table 3.

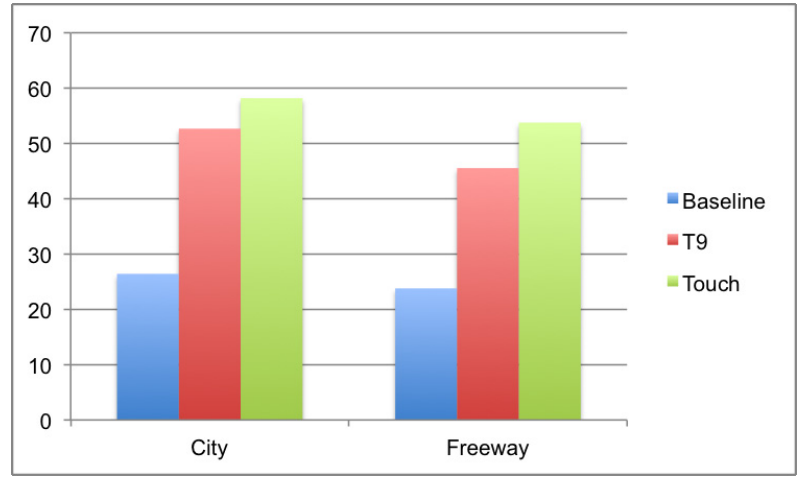

Fig. 7. Task load

Task Load. The subjects' average perceived task load doubled when writing text messages. This was significant both on the freeway $(\mathrm{F}=11.36, \mathrm{p}<0.001)$ and in the city $(\mathrm{F}=17.73, \mathrm{p}<0.001)$. The task load was also slightly higher when writing with Touch than it was when writing with T9, but this difference was found not to be significant. The average perceived task load was slightly higher when driving in the city than it was when driving on the freeway, but this difference was not significant.

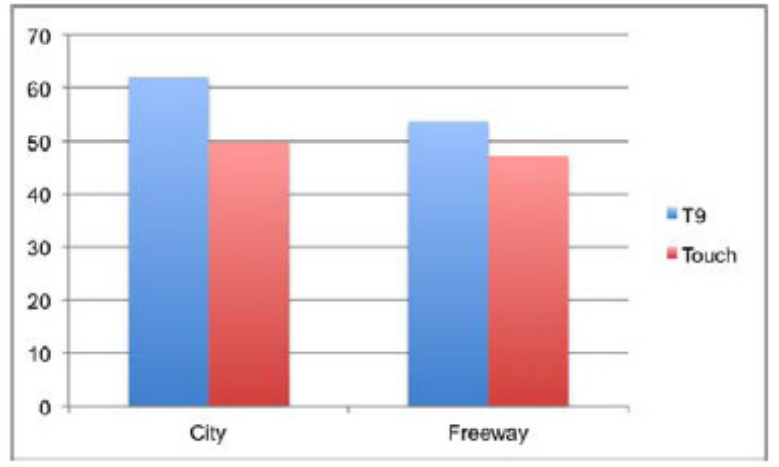

Fig. 8. Average characters per minute

Task Completion. The average number of successfully completed task per subject was higher for T9 than it was for Touch, but this difference was not found to be significant.

Writing Speed. The average number of characters per minute was higher with T9 than it was with Touch, but no significant difference was found. 


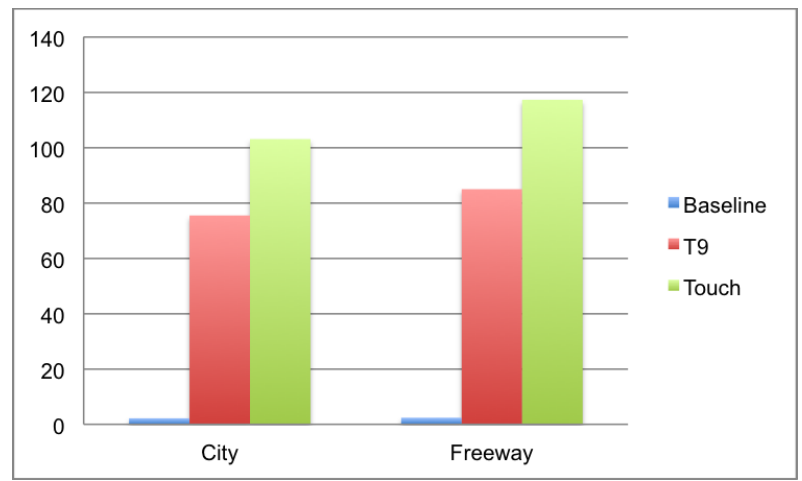

Fig. 9. Average time spent looking away

\subsection{Eye Glances}

The results on eye glances are shown in table 4 . The effect of writing on time spent looking away was significant both on the freeway $(\mathrm{F}=48.81, \mathrm{p}<0.001)$ and in the city $(\mathrm{F}=59.69, \mathrm{p}<0.001)$. There was a significant increase in time spent looking away when writing with Touch compared to T9 both in the city $(\mathrm{F}=5.66, \mathrm{p}<0.05)$ and on the freeway $(\mathrm{F}=4.87, \mathrm{p}<0.05)$. This was caused by a significant increase in category 3 eye glances both in the city $(\mathrm{F}=9.92, \mathrm{p}<0.01)$ and on the freeway $(\mathrm{F}=10.00, \mathrm{p}<0.01)$. There was no significant difference in category 1 and category 2 eye glances between Touch and T9.

Table 4. Means and standard deviations of eye glances

\begin{tabular}{l|ccc} 
& \multicolumn{3}{|c}{ Condition } \\
\hline Variable & Baseline & T9 & Touch \\
\hline City & & & \\
Time spent looking away (s) & $2.18(1.56)$ & $75.52(27.55)$ & $103.14(32.75)$ \\
Category 1 $<0.5$ seconds) & $0.54(0.66)$ & $1.39(2.06)$ & $2.15(1.95)$ \\
Category 2(0.5-2.0 seconds) & $2.54(2.54)$ & $45.31(13.96)$ & $42.31(25.36)$ \\
Category 3 (>2.0 seconds) & $0.00(0.00)$ & $9.00(8.85)$ & $18.69(8.92)$ \\
& & & \\
\hline Freeway & & & \\
Time spent looking away (s) & $2.40(2.23)$ & $85.02(33.57)$ & $117.35(44.59)$ \\
Category 1 $<0.5$ seconds) & $0.14(0.36)$ & $0.50(0.85)$ & $1.07(1.32)$ \\
Category 2 (0.5-2.0 seconds) & $2.79(2.69)$ & $43.57(11.69)$ & $39.00(23.90)$ \\
Category 3 (>2.0 seconds) & $0.00(0.00)$ & $12.07(9.30)$ & $24.07(9.93)$ \\
& & &
\end{tabular}

The results showed no significant difference on time spent looking away between driving in the city and the freeway. 


\subsection{Expert Comparison}

A recent study assessed whether frequent texters are less dangerous in the traffic than less frequent texters. Based on their data, they concluded that this is not the case [34].

Our experiment was not designed to study this aspect. However, for all the results presented in the three tables above, we made between-subject ANOVA tests between T9 users in the T9 condition and Touch users in the Touch condition. The analysis showed no major differences compared to the overall results. This suggests that increased experience with a mobile phone does not change the effect on road safety when texting while driving.

\section{Discussion}

The results on driving performance show a clear change in driving behaviour when writing text messages compared to driving without interacting with a mobile phone. Between the baseline and the two writing conditions, we have significant results showing that the reaction time doubled, risk of crash/near-crash was 4-5 times higher, and the following distance, following distance variability and lane crossings increased in both the city and on the freeway, and so did lane variability on the freeway.

In the overview of related work, we mentioned several studies of mobile use for calling. Our results are consistent with these studies which demonstrate that text messaging is just as dangerous as other uses of a mobile phone.

The combination of reaction time and crash/near-crash risk shows that writing text messages while driving increases the risk of being in a dangerous situation by around 4 times in the city, and around 5 times on the freeway.

For several of our measures, there was a tendency that the smartphone decreased road safety compared to the traditional tactile phone. However, only some of these differences were significant. The main difference for performance was the number of crash or near-crash incidents and the lane crossings which were significant on the freeway. These results show that overall, text messaging with touch-based smartphone is more dangerous than with T9.

This increase in crash and near-crash risk from city to freeway may be caused by the straight and wider road of the freeway, compared to the many curved roads and intersections in the city. Virginia Research [7] found that texting while driving increased the odds of crashes or near crashes by 23 times. We only found an increase of four but this is possibly attributed to the difference in testing environments, as their testing was done in vehicles in real traffic and not in a simulator. Furthermore, our simulator did not have elements such as pedestrians or cyclists, which, if present, could have increased the likelihood of crash and near-crash situations. On the other hand, our users were in the younger end of the scale, and they may be better able to deal with the distraction from the mobile phone.

Our subjects clearly increased their following distance when texting, suggesting that they subconsciously attempted to reduce the likelihood of being involved in a crash. This is consistent with other results [11]. 
We analysed our videos looking for explanations of this difference between city and freeway. In the city, our drivers seemed more stressed because they had to follow a car that was stopping, starting and turning frequently. They also had to watch traffic lights and cars driving in the opposite direction. This seems to increase their attention on the traffic. On the freeway, there is less activity and they are more relaxed, which makes them pay less attention to the driving. This is also consistent with the results on lane variability. Some of the related studies have results indicating that there are fewer crashes or near-crashes in the city.

The results on task performance point in the same direction. The task load doubled from the baseline to the two texting conditions. Thus the subject perceived it to be much more demanding to write text messages while driving. There was a tendency that T9 was faster on task completion and writing speed compared to the Touch phone, but this difference was not significant.

The results on eye glances show that writing messages causes the driver to look away from the traffic more often and for longer periods of time. The drivers spent 30-50 times more time looking away when they were writing text messages. The number of eye glances, where the driver looks away from the traffic, increased significantly. In addition, the increase was on the long (between a half and two seconds) and very long (over 2 seconds) eye glances. Research on road safety has shown that these longer eye glances are much more dangerous than the shorter ones.

When we compare the two mobile telephones, the users with the Touch phone spent significantly more time looking away, and they had the double number of very long eye glances compared to the users of the T9 phone.

The test subjects lane variability, crash/near-crash ratio and time spent in the wrong lane as well as time spent looking away was increased when using a smartphone. This suggests that the lack of tactile feedback on the phones caused the subject to look away for longer periods of time, which is why only the number of category 3 eye glances (above 2 seconds) was significantly higher when using a smartphone. These results confirm our original expectation that the touch-based smartphone requires more visual attention.

Overall, the results confirm that text messaging during simulated driving has significant impact on road safety. On some parameters, there was a tendency that texting on the smartphone reduced road safety even more than the traditional phone. On the more severe parameters, there were significant results showing that the touch phone was more dangerous than the traditional tactile mobile phone.

\section{Conclusion}

In this paper we presented results from an empirical study of the effect of text messaging on road safety where we specifically compared the use of a traditional mobile phone and a smartphone for writing text messages during simulated driving. The results clearly show that writing text messages using any of the two mobile phones significantly decrease road safety. This is caused by significant changes in driving behaviour related to reaction time and lane keeping. Writing text messages while 
driving increased the risk of being involved in a crash or near-crash situation by a factor of 4 to 5 .

The results suggest that while the touch-based smartphone is a step forward in many respects, it decreases road safety when used for writing text messages while driving. The subjects spent significantly more time looking away when they used the smartphone, and this increased the risk of crash or near-crash incidents. Texting while driving is increasing rapidly, and our results show that when a smartphone is used for this, it increases driver distraction as this type of phone provide no tactile feedback, leaving only visual feedback when writing text messages. Our results suggest that drivers should never text while driving, especially not with a smartphone.

There are some limitations in our study. The experiment was conducted in a simulator and not in real traffic. However, our results point in the same direction as studies of real traffic, and comparisons of car simulators and real traffic confirm the relevance of the results from simulators. Our test subjects were relatively young, and, thereby, more experienced in texting. This could imply that they are less dangerous; on the other hand, young people are more likely to be involved in accidents.

For a future study, it could be interesting to identify the factors of mobile phone interaction while driving that affect distraction. Related to this, it would be interesting to further identify the exact points where touch-based smartphones differ from traditional tactile mobile phones. This could include studies with different keyboards and types of feedback on the smartphone.

Acknowledgements. The research behind this paper was partly financed by the Danish Research Councils (grant number 09-065143). We are grateful to the test subjects who participated and the anonymous reviewers.

\section{References}

1. Countries that ban mobile phones while driving, http: / /www. cellularnews.com/car_bans /

2. Car crash statistics based on age and location, http: / /www. autos.com/drivingandsafety/ carcrashstatisticsbasedonageandlocation

3. Alm, H., Nilsson, L.: Changes in driver behaviour as a function of handsfree mobile phones - A simulator study. Accident Analysis and Prevention 26, 441-451 (1994)

4. Alm, H., Nilsson, L.: The effects of a mobile telephone task on driver behaviour in a car following situation. Accident Analysis and Prevention 27, 707-715 (1995)

5. Arif, A.S., Lopez, M.H., Stuerzlinger, W.: Two new mobile touchscreen text entry techniques. In: 36th Graphics Interface Conference (2010)

6. Battestini, A., Setlur, V., Sohn, T.: A large scale study of text messaging use. In: MobileHCI (2010)

7. Box, S.: New data from VTTI provides insight into mobile phone use and driving distraction. Driving Transportation with Technology (2009)

8. Brookhuis, K.A., De Vries, G., De Waard, D.: The effects of mobile telephoning on driving performance. Accident Analysis and Prevention 23, 309-316 (1991) 
9. Charlton, S.G.: Driving while conversing: Mobile phones that distract and passengers who react. Accident Analysis and Prevention 41, 160-173 (2009)

10. Company, T.N.: How teens use media. A Nielsen report on the myths and realities of teen media trends (2009)

11. Curran, K., Woods, D.: Investigating text input methods for mobile phones. Telemat. Inf. 23(1), 1-21 (2006)

12. Drews, F.A., Yazdani, H., Godfrey, C.N., Cooper, J.M., Strayer, D.L.: Text messaging during simulated driving. Human Factors (2009)

13. Entner, R.: Smartphones to overtake feature phones in u.s. by 2011. The Nielsen Company (2010)

14. Favalora, L.: Texting and driving kills. Dr. Frank Ryan's death while texting and driving is a warning. Global Press Release Distribution (2010)

15. Foundation, A.: Text messaging and mobile phone use while driving. Safety Culture (2009)

16. Gartner. Mobile Phone Sales Q3 (2010), http: / / www.gartner.com/it/page.jsp?id=1466313

17. Gilbert, J.E., Martin, A.M., Eugene, W., Alnizami, H., Moses, W., Morrison, D.: Driving transportation policy through technological innovation. Brave New World (2010)

18. Hart, S.G.: Nasa-task load index (nasa-tlx); 20 years later. NASA-Ames Research (2006)

19. Hosking, S.G., Young, K.L.: The effects of text messaging on young drivers. Human Factors (2009)

20. James, C.L., Reischel, K.M.: Text input for mobile devices: comparing model prediction to actual perfor-mance. In: Proceedings of CHI 2001, pp. 365-371. ACM (2001)

21. Jensen, B.S., Skov, M.B., Thiruravichandran, N.: Studying driver attention and behaviour for three configurations of GPS navigation in real traffic driving. In: Proceedings of CHI 2010, pp. 1271-1280. ACM (2010)

22. Joergensen, M.: Mobiltelefoner og bilkoersel. Trafik og Veje (2010)

23. Kircher, A., Vogel, K., Tornros, J., Bolling, A., Patten, N.L.C., Malmstrom, T., Ceci, R.: Mobile telephone simulator study. Swedish National Road and Transport Research Institute. Swedish National Road and Transport Research Institute (2004)

24. Klauer, S., Dingus, T.A., Neale, V.L., Sudweeks, J., Ramsey, D.: The impact of driver inattention on near-crash/crash risk. Technical report, U.S. Department of Transportation (2006)

25. Kristiansen, A.H., Larsen, F.: QdQ: A new keyboard for writing text on small soft keyboards. Master Thesis, Aalborg University (2009)

26. Lee, J.D., Strayer, D.L.: Preface to the special section of driver distraction. Human Factors (2004)

27. McEvoy, S., Stevenson, M.R., McCartt, A.T., Woodward, M., Haworth, C., Palamara, P., Cercarelli, R.: Role of mobile phones in motor vehicle crashes resulting in hospital attendance: a case-crosover study. BMJ 331 (2005)

28. McKnight, A.J., McKnight, A.S.: The effect of cellular phone use upon driver attention. Accident Analysis and Prevention 25, 259-265 (1993)

29. McPhee, L.C., Scialfa, C.T., Dennis, W.M., Ho, G., Caird, J.K.: Age differences in visual search for traffic signs during a simulated conversation. Human Factors (2004)

30. Olswang. Convergence Survey (2011), http: / /www. olswang.com/convergence2011/

31. Redelmeier, D.A., Tibshirani, R.J.: Association between cellular-telephone calls and motor vehicle collisions. New England Journal of Medicine 336, 453-458 (1997) 
32. Reed, M.P., Green, P.A.: Comparison of driving performance on-road and in a lowcost simulator using a concurrent telephone dialing task. Ergonomics 42, 1015-1037 (1999)

33. Salvucci, D.D., Markley, D., Zuber, M., Brumby, D.P.: iPod Distraction: Effects of Portable Music-Player Use on Driver Performance. In: Proceedings of CHI 2007, pp. 243-250. ACM (2007)

34. Samuel, S., Pollatsek, A., Fisher, D.: Texting While Driving: Evaluation of Glance Distributions for Frequent/Infrequent Texters and Keypad/Touchpad Texters. In: Proceedings of the Sixth International Driving Symposium on Human Factors in Driver Assessment, Training and Vehicle Design (2008)

35. Strayer, D.L., Drews, F.A., Crouch, D.J.: Fatal distraction? A comparison of the cell-phone driver and the drunk driver. University of Utah Department of Psychology (2003)

36. Wood, C., Hurwitz, J.: Driver workload management during mobile phone conversations. In: Driving Assessment 2005: Proceedings of the 3rd International Symposium on Human Factors in Driver Assessment, Training, and Vehicle Design (2005) 